\title{
A reference map of the Arabidopsis thaliana mature pollen proteome ${ }^{i s}$
}

\author{
Sandra Noir, Anne Bräutigam, Thomas Colby, Jürgen Schmidt, Ralph Panstruga * \\ Max-Planck-Institute for Plant Breeding Research, Carl-von-Linné-Weg 10, D-50829 Köln, Germany
}

Received 15 September 2005

Available online 7 October 2005

\begin{abstract}
The male gametophyte (or pollen) plays an obligatory role during sexual reproduction of higher plants. The extremely reduced complexity of this organ renders pollen a valuable experimental system for studying fundamental aspects of plant biology such as cell fate determination, cell-cell interactions, cell polarity, and tip-growth. Here, we present the first reference map of the mature pollen proteome of the dicotyledonous model plant species, Arabidopsis thaliana. Based on two-dimensional gel electrophoresis, matrix-assisted laser desorption/ionization time-of-flight, and electrospray quadrupole time-of-flight mass spectrometry, we reproducibly identified 121 different proteins in 145 individual spots. The presence, subcellular localization, and functional classification of the identified proteins are discussed in relation to the pollen transcriptome and the full protein complement encoded by the nuclear Arabidopsis genome.
\end{abstract}

(c) 2005 Elsevier Inc. All rights reserved.

Keywords: Arabidopsis thaliana; Male gametophyte; Mass spectrometry; Mature pollen; Proteome; Two-dimensional gel electrophoresis

In higher plants, development of the male gametophyte is a well-programmed and elaborate process. Male sporogenesis begins with the division of a diploid sporophytic cell giving rise to the anther wall of the stamen and the sporogenic cells. The latter cells then undergo several mitoses to differentiate into pollen mother cells. Subsequently, each diploid pollen mother cell forms a tetrad of haploid microspores via a round of DNA replication followed by two consecutive meiotic divisions. Each uninucleate microspore then undergoes an asymmetric mitotic division forming a large vegetative cell and a smaller generative cell (i.e., the bicellular pollen stage). In Arabidopsis, the generative cell undergoes another mitotic division giving rise to two sperm cells (i.e., the tricellular pollen stage). Following pollination, the vegetative cell controls the further develop-

\footnotetext{
Abbreviations: 2-DE, two-dimensional gel electrophoresis; Chaps, 3-(3cholamidopropyl)dimethylammonio-1-propane sulfonate; DAPI, 4',6-diamino-phenylindole; FDA, fluorescein diacetate; IEF, isoelectric focusing; LC-MS/MS, liquid chromatography coupled to tandem mass spectrometry; MALDI-TOF, matrix-assisted laser desorption/ionization time-offlight; MS, mass spectrometry; $\mathrm{p}$, isoelectric point; PMF, peptide mass fingerprint.

* Corresponding author. Fax: +49 2215062353

E-mail address: panstrug@mpiz-koeln.mpg.de (R. Panstruga).
}

ment of the mature pollen grain and growth of the pollen tube into the style until both sperm cell nuclei are delivered to the embryo sac in the ovule, where they participate in double fertilization $[1,2]$.

The last two decades have been marked by increasing efforts to decipher the genetic and molecular basis of pollen development and functions [reviewed in 1,3,4]. In the model plant Arabidopsis thaliana, the extremely reduced, tricellular male gametophyte constitutes an ideal experimental system for analyses of important biological processes in higher plant reproduction. In addition, it represents a very useful model for studying fundamental aspects of plant biology such as cell fate determination, cell-cell interactions, cell polarity, and tip-growth [5-7].

The availability of the full genome sequence of Arabidopsis [8] has made genome-wide, microarray-based analyses of the male gametophyte transcriptome of this model plant possible [9-12]. 13,977 male gametophyte-expressed mRNAs were recently identified using the ATH1 Genome Array, which covers $\sim 81 \%$ of the currently estimated 28,000 protein-coding genes in Arabidopsis. It seems that $9.7 \%$ of these transcripts are specific for the male gametophyte [11]. The majority of the respective genes appear to be expressed during the two earliest developmental stages 
of sporogenesis (i.e., in microspores and bicellular pollen). Indeed, 8788 transcripts were found to be expressed in tricellular pollen [11] and either 7235 [11] or 6587 [12] in mature pollen. Compared to the uninucleate microspore stage, male-gametophyte-specific genes identified from the mature pollen grain stage exhibit an increase in the collective proportion of cell wall-, cytoskeleton-, signaling-, and transport-related genes [11]. This rise is thought to reflect the functional specialization of mature pollen in preparation for a dramatic change in its cell growth pattern during pollen germination and pollen tube growth.

Although global gene expression analysis is useful for selecting candidates for functional studies, the presence or absence of a given transcript does not necessarily have predictive power for the presence or absence of the protein it encodes. For example, minute levels of a transcript may be sufficient for adequate accumulation of the respective protein, while post-transcriptional and/or post-translational regulation might negatively affect accumulation of particular proteins encoded by well-represented transcripts. Thus, in addition to transcriptome analyses, it is equally important to determine the protein complement of a given tissue. To this end, a two-dimensional gel electrophoresis (2-DE)based proteomic approach has already been applied to study the differential protein synthesis and protein phosphorylation patterns of Brassica napus microspores during microspore embryogenesis [13] and to compare pollen coat protein profiles of self-incompatible and self-fertile Festuca pratensis [14]. In addition, to develop information about the proteins present at different stages of pollen development, 2DE and mass spectrometry have been used to analyze the protein complement of rice anthers $[15,16]$. In this study, the authors were able to identify $\sim 150$ protein spots that varied among different stages of anther development. So far, the only reported proteome analysis in A. thaliana described the constituents of the pollen coat [17]. The work presented here reports the application of proteome analysis techniques to characterize the Arabidopsis mature pollen stage. Using 2-DE in combination with MALDI-TOF MS and LC-MS/MS analyses, we generated the first reference map of the Arabidopsis mature pollen proteome.

\section{Materials and methods}

Plant material and pollen isolation. Plants of $A$. thaliana Columbia (Col-0) ecotype were grown in the greenhouse at $20^{\circ} \mathrm{C}$-day and $18{ }^{\circ} \mathrm{C}$ night and $50 \%$ relative humidity, under $8 \mathrm{~h}$ light $/ 16 \mathrm{~h}$ dark for the 3 first weeks, and then $16 \mathrm{~h}$ light $/ 8 \mathrm{~h}$ dark regimes until flowering. In addition to wild-type plants, three homozygous double knockout (T-DNA or transposon insertion) lines in genes AtMlo5 and AtMlo9 were employed in the experiments. These lines are homozygous $\mathrm{F}_{2}$ progeny from crosses of insertion lines SLAT_15-21 (Atmlo5-2) and GARLIC_348_B04 (Atmlo92), SALK_118934 (Atmlo5-5) and SALK_073198 (Atmlo9-3), as well as SLAT_24-28 (Atmlo5-1) and GARLIC_348_B04 (Atmlo9-2), respectively. Sequence-indexed SALK [18] and GARLIC (now referred to as SAIL) [19] T-DNA insertion lines were obtained from the Nottingham Arabidopsis Stock Centre (NASC; http://arabidopsis.info/) and Syngenta Biotechnology, respectively. SLAT transposon insertion lines [20] were selected by PCR using pooled DNA samples and appropriate oligonucleotides. Ma- ture pollen grains from $\sim 400$ plants per genotype were harvested using a vacuum system adapted from Johnson-Brousseau and McCormick [21], and stocked at $-20^{\circ} \mathrm{C}$ until protein extraction (see below).

Pollen purity and viability were assessed using representative samples of the frozen pollen batches. The purity of isolated pollen was determined by light microscopy and epifluorescence microscopy after 4',6-diaminophenylindole (DAPI, Sigma) staining $(2.5 \mu \mathrm{g} / \mathrm{ml}$ DAPI in $50 \mathrm{mM}$ PBS, $\mathrm{pH}$ 7.2 , with $0.01 \%$ Tween 20 and $5 \%$ DMSO). Samples were observed via epifluorescence $1 \mathrm{~h}$ after incubation in DAPI solution at room temperature. Pollen viability was analyzed $2 \mathrm{~h}$ after rehydration at $4{ }^{\circ} \mathrm{C}$ in a high humidity chamber and staining with fluorescein diacetate (FDA, Fluka). A stock solution of $2 \mathrm{mg} / \mathrm{ml} \mathrm{FDA} \mathrm{was} \mathrm{made} \mathrm{in} \mathrm{acetone} \mathrm{and} \mathrm{diluted} \mathrm{drop-by-}$ drop in $0.5 \mathrm{M}$ of sucrose until the solution remained milky. Samples were observed by epifluorescence $5 \mathrm{~min}$ after incubation at room temperature.

Protein extraction. Pollen grains were suspended in $600 \mu \mathrm{l}$ of extraction buffer (1 protease inhibitor cocktail tablet (Roche); $10 \mathrm{mM}$ DTT; $50 \mathrm{mM}$, $\mathrm{pH}$ 8.0, Tris-Base; $10 \mathrm{mM}$ EDTA; and $0.5 \%$ Chaps) and a small amount of glass beads (diameters of 425-600 $\mu \mathrm{m}$, Sigma) were added. Pollen grains were broken by five repetitions of vortexing ( $30 \mathrm{~s})$ interrupted by short pauses on ice ( $30 \mathrm{~s}$ each). After centrifugation at $16,000 \mathrm{~g}$ for $5 \mathrm{~min}$ at $4{ }^{\circ} \mathrm{C}$, the supernatant was removed and stored on ice. From the remaining pellet, the extraction procedure was repeated and supernatants were pooled. Protein concentration of the supernatant was determined by the Bradford assay with bovine serum albumin as the standard. Supernatants were dried frozen and stored at $-80{ }^{\circ} \mathrm{C}$ until loading for isoelectric focusing.

Two-dimensional gel electrophoresis. Two-dimensional polyacrylamide gel electrophoresis (2-DE) was performed using the NuPAGE ZOOM Benchtop Proteomics system (Invitrogen). Proteins $(100 \mu \mathrm{g})$ were solubilized in $165 \mu \mathrm{l}$ sample rehydration buffer ( $7 \mathrm{M}$ urea, $2 \mathrm{M}$ thiourea, $2 \%$ Chaps, 0.5\% ZOOM Carrier Ampholytes, pH 3-10 (Invitrogen), $20 \mathrm{mM}$ DTT, and $0.1 \%$ bromophenol blue). Prior to isoelectric focusing (IEF) ZOOM strips, pH 3-10, NL (Invitrogen) were incubated in the rehydration solution containing the sample for $16 \mathrm{~h}$, and IEF was conducted using the following step gradient: $0-175 \mathrm{~V}(1 \mathrm{~min}), 175 \mathrm{~V}(15 \mathrm{~min}), 175-2000 \mathrm{~V}$ (45 min), and $2000 \mathrm{~V}$ (25 min). After IEF, the strips used for gel electrophoresis were first equilibrated in $4.5 \mathrm{ml}$ lithium dodecyl sulfate (LDS) sample buffer together with $0.5 \mathrm{ml}$ of $10 \times$ sample reducing agent (Invitrogen), and subsequently in the same solution containing $125 \mathrm{mM}$ iodoacetamide without reducing agent (15 min each). Samples were separated in the second dimension on NuPAGE Novex $4-12 \%$ Bis-Tris ZOOM gels in Mes-SDS running buffer (Invitrogen), and proteins were stained with colloidal Coomassie using Imperial Protein Stain (Pierce).

In-gel digestion and mass spectrometry. After 2-DE, spots of various intensities were automatically picked (PROTEINEERsp, Bruker) and tryptically digested using the DP Chemical 96 Kits for fully automated ingel digestion (PROTEINEERdp, Bruker). Aliquots of the digests were automatically prepared (PROTEINEERdp, Bruker) for subsequent MALDI-TOF analysis on AnchorChip targets (Bruker) according to Gobom et al. [22]. Mass spectra of tryptic peptides were taken with a Bruker Reflex IV MALDI-TOF MS. The obtained peptide mass fingerprints (PMFs) were processed in Xmass 5.1.16 (Bruker) and used to identify the corresponding proteins in the ProteinScape 1.2 database system (Protagen AG) which triggered Mascot (Matrix Science) and ProFound (Genomic Solutions) searches. Sample aliquots of spots containing several putative proteins were submitted to in-depth LC-MS/MS analyses.

$L C-M S / M S$ analysis. Residual organic solvent was removed from sample digests via vacuum centrifugation prior to injection into a Waters CapLC coupled to a Q-ToFII mass spectrometer (Micromass/Waters). Samples were loaded onto a Waters Symmetry 300 c18 trapping column for on-column focusing and eluted over a Waters Atlantis c18 analytical column $(75 \mu \mathrm{m} \times 150 \mathrm{~mm})$ with a $5-50 \% \mathrm{ACN}$ gradient containing $0.1 \%$ formic acid. The elution was analyzed in survey mode under the control of Masslynx4.0 (Waters). Multiply charged peaks of sufficient intensity were automatically fragmented in the argon collision cell and the resulting MS/ MS spectra were processed in Masslynx4.0 before submission to Mascot (Matrix Science) for searches against the NCBI non-redundant database.

Pollen transcriptome analysis. The pollen transcriptome analysis has been performed using Affymetrix GeneChip technology. Two independent 
experiments have been carried out using Col-0 wild-type plants and the three Atmlo5/Atmlo9 double mutant lines. Mature pollen grains from $\sim 400$ plants each per genotype were harvested using the above-mentioned vacuum system. Total RNAs were extracted using the RNeasy Plant Mini Kit (Qiagen) and used for Arabidospsis GeneChip ATH1 array hybridizations (University of Cologne-Germany) under standard conditions [23]. MicroArray Suite 5.0 software was used for data analysis.

Database searching. The PMF data were used to screen the National Center for Biotechnology Information (NCBI) database (http:// www.ncbi.nlm.nih.gov/entrez/query.fcgi?db=Protein). When AGI names could not be identified, data were completed by using the TAIR BLAST 2.2.8 tool. The TAIR database (http://www.arabidopsis.org/index.jsp) was also used to annotate identified proteins and to assign their predicted subcellular localization. The latter were corroborated by using the pSORT Wolf software for Protein Subcellular Localization Prediction (http:// wolfpsort.seq.cbrc.jp/). Potential transmembrane domains were investigated by using the TAIR Bulk Protein Search Tool. Functional categories were assigned according to the Functional Catalogue Database of MIPS (http://mips.gsf.de/projects/funcat). When multiple functional categories were allotted to an individual protein, the most probable (i.e., lowest $p$ value) was chosen. Arabidopsis organs where identified genes are preferentially expressed were deduced by using the Gene Atlas tool of Genevestigator (https://www.genevestigator.ethz.ch/) [24].

\section{Results and discussion}

The results of this study were obtained in the context of a comparative proteome analysis between A. thaliana Col-0 wild-type plants and Atmlo5/Atmlo9 double knockout mutants in the Col-0 genetic background (see Materials and methods for details). Genes AtMlo5 and AtMlo9 encode two members of the family of heptahelical MLO membrane proteins [25] that are preferentially expressed during pollen development [Z.Y. Chen and co-workers, personal communication; S. Noir and R. Panstruga, unpublished]. In the context of this report, we take advantage of the fact that no differences between the proteomes of mature pollen from wild-type plants and Atmlo5/Atmlo9 knockout mutants could be observed and employ the respective dataset for the establishment of the first reference map of the Arabidopsis mature pollen proteome.

\section{Mining of pollen source material}

Approximately $20 \mathrm{mg}$ of mature pollen each was collected from $\sim 400$ plants per genotype (wild-type plus three distinct Atmlo5/Atmlo9 double mutants). Microscopic examination of pollen populations after DAPI staining confirmed the homogeneity of these samples (i.e., no other developmental stage besides mature tricellular pollen was observed). Little cellular debris was detected and FDA staining revealed that the proportion of viable pollen grains was $\sim 75 \%$ (data not shown). From each pollen sample, $\sim 1800-2000 \mu \mathrm{g}$ of total protein could be extracted.

\section{Establishment of a proteome map of Arabidopsis mature pollen}

Aliquots $(\sim 100 \mu \mathrm{g}$ each) of the protein samples were subjected to 2-DE as described in Materials and methods. Analysis of at least two replicate gels of the four distinct $A$. thaliana genotypes revealed no reproducible differences between samples derived from Col-0 wild-type plants and Atmlo5/Atmlo9 double mutants (data not shown). In total, up to 145 mature pollen protein spots could be reproducibly resolved over a $\mathrm{p} I$ range of 3-10 on Coomassie bluestained gels (Fig. 1).

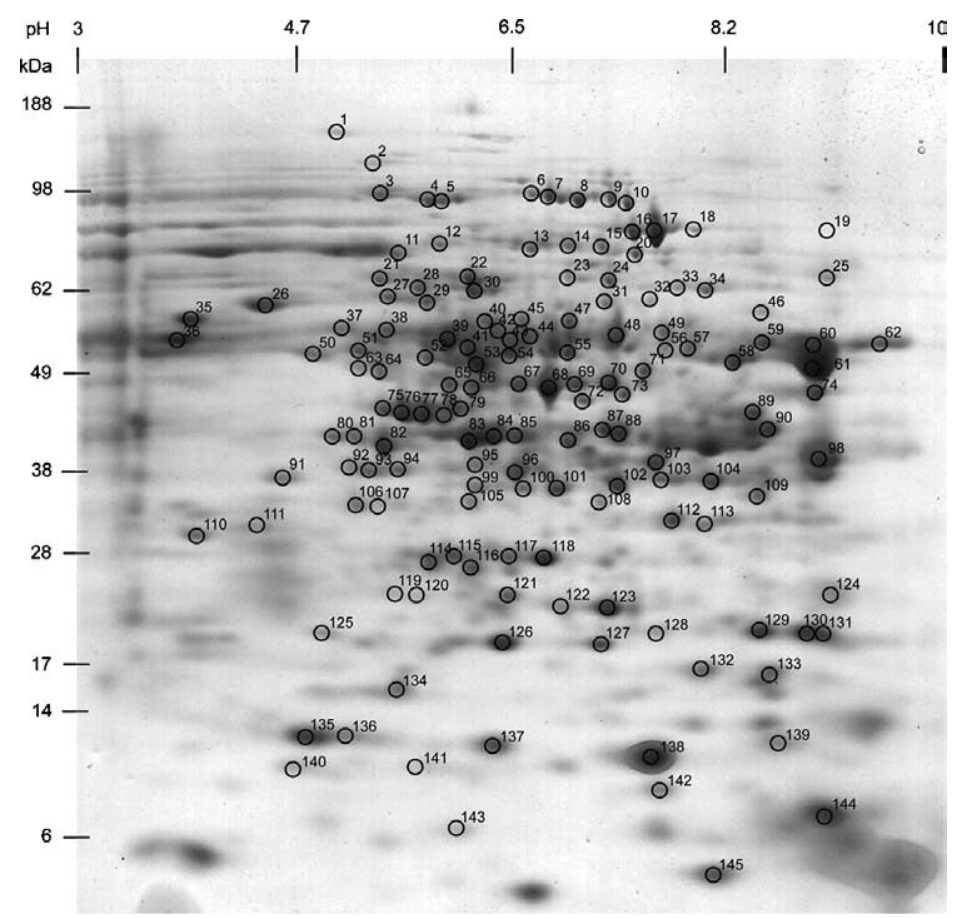

Fig. 1. Coomassie blue-stained two-dimensional gel of a total protein extract $(100 \mu \mathrm{g})$ from mature pollen of Arabidopsis. Molecular masses are given in $\mathrm{kDa}$ and approximate isoelectric points are shown. The identification of each labeled spot can be found in Table 1 . 
Table 1

Results of protein identification in Arabidopsis mature pollen

\begin{tabular}{|c|c|c|c|c|c|c|c|c|c|c|c|}
\hline Spot No. ${ }^{a}$ & AGI name & Gene index & Protein identity (TAIR description) & $\mathrm{MM} / \mathrm{p} I^{\mathrm{b}}$ & $\begin{array}{l}\text { Sequence } \\
\text { coverage }^{\mathrm{c}}\end{array}$ & ProFound-score $^{\mathrm{d}}$ & Mascot-score $^{e}$ & $\begin{array}{l}\text { Subcellular } \\
\text { localization }^{\mathrm{f}}\end{array}$ & $\begin{array}{l}\text { Functional } \\
\text { category }^{\mathrm{g}}\end{array}$ & Genevestigator $^{\mathrm{h}}$ & $\begin{array}{l}\text { Micro array } \\
\text { detection }^{\mathrm{i}}\end{array}$ \\
\hline 1 & Atlg49490 & gi|5430752 & $\begin{array}{l}\text { Leucine-rich repeat family protein/extensin } \\
\text { family protein }\end{array}$ & $90.9 / 4.8$ & 17.7 & 2.4 & 131 & vacuole & unc. & 1 & $\mathrm{P}$ \\
\hline 2 & At2g41740 & gi|22136974 & Villin 2 & $107.8 / 5.1$ & 24.1 & 1.5 & 146 & nucleus & cell wall & 2 & $\mathrm{P}$ \\
\hline 3 & At3g09840 & gi|6681343 & Cell division cycle protein 48 & $89.3 / 5.0$ & 50.7 & 2 & 329 & endomb. & prot. proc. & 3 & $\mathrm{P}$ \\
\hline 4 & At4g15530 & gi|7268300 & Pyruvate phosphate dikinase family protein & $104.9 / 5.8$ & 31.3 & 2.3 & 273 & cytp. & energy & 2 & $\mathrm{P}$ \\
\hline 5 & At4g15530 & gi|7268300 & Pyruvate phosphate dikinase family protein & $104.9 / 5.8$ & 34.5 & 2.2 & 302 & cytp. & energy & 2 & $\mathrm{P}$ \\
\hline 6 & At4g26970 & gi|23308183 & Putative aconitate hydratase & 108.3/7.0 & 36.0 & 2.2 & 293 & endomb. & energy & 2 & $\mathrm{P}$ \\
\hline 7 & At4g26970 & gi|23308183 & Putative aconitate hydratase & $108.3 / 7.0$ & 43.4 & 1.7 & 307 & endomb. & energy & 2 & $\mathrm{P}$ \\
\hline 8 & At2g05710 & gi|4586021 & Aconitate hydratase & $98.1 / 5.7$ & 59.0 & 2.4 & 472 & cytp. & energy & 3 & $\mathrm{P}$ \\
\hline 9 & At4g35830 & gi|7270535 & Aconitate hydratase & $98.1 / 6.0$ & 45.1 & 2 & 305 & cytp. & energy & 3 & $\mathrm{P}$ \\
\hline 10 & Atlg56075 & gi|23397162 & Putative elongation factor 2 & $93.8 / 5.9$ & 53.7 & 2.4 & 318 & cytp. & unc. & no data & no data \\
\hline 11 & At5g09590 & gi|7671430 & Heat shock protein 70 & $72.9 / 5.5$ & 53.7 & 2.2 & 230 & endomb. & defense & 3 & undeter. \\
\hline 12 & At3g60750 & gi|22136900 & Putative transketolase & $79.9 / 5.9$ & 39.7 & 2 & 228 & endomb. & energy & 4 & undeter. \\
\hline 13 & At2g45290 & gi|20196914 & Putative transketolase & $68.8 / 5.6$ & 43.4 & 1.8 & 214 & endomb. & energy & 2 & undeter. \\
\hline $14^{* *}$ & At2g07698 & gi|14916970 & Putative ATP synthase alpha chain & $55.3 / 6.2$ & j & j & 94 & endomb. & energy & 3 & A \\
\hline $14^{* *}$ & At3g24170 & gi|14532810 & Putative glutathione reductase & $54.3 / 6.4$ & j & $\mathrm{j}$ & 64 & cytp. & unc. & 4 & undeter. \\
\hline 15 & At5g65690 & gi|10177326 & $\begin{array}{l}\text { Putative phosphoenolpyruvate } \\
\text { carboxykinase }\end{array}$ & $68.7 / 6.0$ & 44.3 & 1.9 & 222 & unknown & energy & no data & no data \\
\hline 16 & At5g17920 & gi|20147149 & Homocysteine methylase & $84.3 / 6.1$ & 45.4 & 2.4 & 295 & cytp. & metabolism & no data & no data \\
\hline 17 & At5g17920 & gi|20147149 & Homocysteine methylase & $84.3 / 6.1$ & 65.8 & 2.4 & 404 & cytp. & metabolism & no data & no data \\
\hline 18 & At5g17920 & gi|20147149 & Homocysteine methylase & $84.3 / 6.1$ & 55.0 & 2.4 & 328 & cytp. & metabolism & no data & no data \\
\hline 19 & At5g17920 & gi|20147149 & Homocysteine methylase & $84.3 / 6.1$ & 38.2 & 2.3 & 214 & cytp. & metabolism & no data & no data \\
\hline 20 & Atlg53500 & gi|33090264 & $\begin{array}{l}\text { NAD-dependent epimerase/dehydratase } \\
\text { family protein }\end{array}$ & $75.2 / 6.0$ & 19.8 & 2.4 & 121 & unknown & unc. & 1 & $\mathrm{P}$ \\
\hline 21 & Atlg78900 & gi|30725440 & Vacuolar ATP synthase catalytic subunit A & $68.8 / 5.0$ & 57.0 & 2.3 & 329 & cytp. & transport & 3 & $\mathrm{P}$ \\
\hline 22 & At5g66760 & gi|21700795 & Succinate dehydrogenase flavoprotein subunit & $69.6 / 5.8$ & 68.0 & 2.4 & 392 & endomb. & energy & 2 & $\mathrm{P}$ \\
\hline 23 & Atlg23190 & gi|20148521 & Phosphoglucomutase & $63.1 / 5.9$ & 39.8 & 2.4 & 210 & cytp. & energy & 2 & $\mathrm{P}$ \\
\hline 24 & Atlg23190 & gi|20148521 & Phosphoglucomutase & $63.1 / 5.9$ & 56.3 & 2.2 & 353 & cytp. & energy & 2 & $\mathrm{P}$ \\
\hline 25 & Atlg67290 & gi|28394059 & Glyoxal oxidase-related & $67.7 / 9.6$ & 59.5 & 2.4 & 354 & endomb. & metabolism & 1 & $\mathrm{P}$ \\
\hline 26 & Atlg21750 & gi|17104689 & Disulfide isomerase-like protein & $55.6 / 4.7$ & 66.7 & 2.4 & 458 & endomb. & unc. & 3 & undeter. \\
\hline 27 & At3g23990 & gi|34098917 & Chaperonin & $61.2 / 5.5$ & 60.0 & 2.4 & 344 & endomb. & prot. proc. & 3 & A \\
\hline 28 & At3g54440 & gi|30523396 & Glycoside hydrolase family 2 protein & $68.4 / 5.1$ & 34.3 & 2.3 & 282 & nucleus & metaC & no data & no data \\
\hline 29 & At2g33210 & gi|2924773 & Putative chaperonin & $55.2 / 5.2$ & 46.9 & 1.5 & 174 & endomb. & prot. proc. & 3 & A \\
\hline 30 & At3g08590 & gi|21280833 & $\begin{array}{l}\text { Putative 2,3-biphosphoglycerate-independent } \\
\text { phosphoglycerate mutase }\end{array}$ & $60.7 / 5.5$ & 65.0 & 1.6 & 286 & cytp. & unc. & 3 & undeter. \\
\hline 31 & At3g11830 & gi|6671939 & Putative chaperonin & $59.7 / 6.0$ & 40.2 & 2.4 & 209 & cytp. & cell cycle & 3 & undeter. \\
\hline 32 & At3g48990 & gi|20799715 & $\begin{array}{l}\text { AMP-dependent synthetase and ligase } \\
\text { family protein }\end{array}$ & $55.5 / 6.1$ & 18.3 & 1.5 & 87 & unknown & metabolism & 3 & $\mathrm{P}$ \\
\hline 32 & At5g42740 & gi|11094242 & Glucose-6-phosphate isomerase & $61.5 / 6.2$ & 44.7 & 2.4 & 204 & unknown & energy & 3 & A \\
\hline 33 & At5g25880 & gi|5107826 & Putative malate oxidoreductase & $64.6 / 6.6$ & 27.9 & 2.4 & 170 & cytp. & energy & 1 & $\mathrm{P}$ \\
\hline 34 & At5g 25880 & gi|5107826 & Putative malate oxidoreductase & $64.6 / 6.6$ & 59.4 & 2.4 & 264 & cytp. & energy & 1 & $\mathrm{P}$ \\
\hline 35 & Atlg56340 & gi|30725696 & Calreticulin 1 & $48.5 / 4.3$ & 35.8 & 2.4 & 104 & endomb. & cell fate & 3 & undeter. \\
\hline 36 & Atlg09210 & gi|16974341 & Calreticulin 2 & $48.1 / 4.2$ & 31.6 & 2.4 & 114 & endomb. & unc. & 2 & undeter. \\
\hline 37 & At4g38510 & gi|7270834 & Putative vacuolar ATP synthase subunit B & $54.3 / 4.9$ & 51.1 & 2.2 & 210 & vacuole & transport & 1 & $\mathrm{P}$ \\
\hline 38 & At3g13930 & gi|23397124 & Putative dihydrolipoamide $S$-acetyltransferase & $58.4 / 8.6$ & 52.1 & 2.1 & 258 & endomb. & energy & 2 & undeter. \\
\hline 39 & At5g08690 & gi|17939849 & ATP synthase $\beta$-chain 2 & $63.3 / 6.6$ & 66.6 & 2.4 & 413 & endomb. & energy & 3 & $\mathrm{P}$ \\
\hline 40 & At5g08690 & gi|17939849 & ATP synthase $\beta$-chain 2 & $63.3 / 6.6$ & 27.2 & 0.9 & 67 & endomb. & energy & 3 & $\mathrm{P}$ \\
\hline 41 & At5g08690 & gi|17939849 & ATP synthase $\beta$-chain 2 & $63.3 / 6.6$ & 42.1 & 2.3 & 145 & endomb. & energy & 2 & $\mathrm{P}$ \\
\hline 42 & At4g34200 & gi|23297595 & Putative D-3-phosphoglycerate dehydrogenase & $63.3 / 6.2$ & 44.3 & 2.3 & 179 & endomb. & energy & 3 & undeter. \\
\hline 43 & At4g13940 & gi|20148279 & Adenosylhomocysteinase & $53.3 / 5.6$ & 49.7 & 2.1 & 256 & cytp. & metabolism & 3 & $\mathrm{P}$ \\
\hline 44 & At5g17310 & gi|14532836 & Putative UTP-glucose-1-phosphate & $51.9 / 5.6$ & 70.0 & 2.1 & 279 & endomb. & metaC & 2 & $\mathrm{P}$ \\
\hline
\end{tabular}




\begin{tabular}{|c|c|c|c|c|c|c|}
\hline 45 & At5g20890 & gi|21537317 & Putative chaperonin & $57.2 / 5.5$ & 68.1 & 2 \\
\hline 46 & At3g18190 & gi|20466822 & Putative chaperonin & $57.7 / 8.7$ & 39.0 & 2.4 \\
\hline 47 & At3g02090 & gi 6513923 & Putative peptidase beta-subunit & $59.1 / 6.3$ & 55.9 & 1.4 \\
\hline 48 & At2g07698 & gi|14916970 & Putative ATP synthase alpha-chain & $55.0 / 6.2$ & 45.6 & 2.4 \\
\hline 49 & At5g65690 & gi|10177326 & Putative phosphoenolpyruvate carboxykinase & $68.7 / 6.0$ & 44.3 & 1.9 \\
\hline 50 & At5g44340 & gi|16323374 & Tubulin $\beta$-4 chain & $49.8 / 4.6$ & 65.3 & 2.4 \\
\hline 51 & Atlg50010 & gi|23506129 & Tubulin $\alpha-2 / \alpha-4$ chain & $49.5 / 4.8$ & 71.3 & 2.4 \\
\hline 52 & Atlg51980 & gi|20258957 & Putative peptidase alpha subunit & $54.4 / 5.9$ & 29.0 & 1.9 \\
\hline 53 & At2g36530 & gi|23297411 & Enolase & $47.7 / 5.5$ & 77.0 & 2 \\
\hline 54 & At5g15490 & gi|53749198 & Putative UDP-glucose 6-dehydrogenase & $53.1 / 5.7$ & 64.6 & 2.2 \\
\hline 55 & At5g15490 & gi|53749198 & Putative UDP-glucose 6-dehydrogenase & $53.1 / 5.7$ & 65.0 & 1.9 \\
\hline $56^{* *}$ & At5g 34850 & gi|20259673 & Calcineurin-like phosphoesterase family protein & $55.0 / 6.9$ & j & \\
\hline 57 & At4g13930 & gi|20334774 & Putative glycine hydroxymethyltransferase & $51.7 / 7.0$ & 32.1 & 1.9 \\
\hline 58 & At2g47510 & gi|2529676 & putative fumarate hydratase & $53.0 / 9.1$ & 46.5 & 1.3 \\
\hline 58 & At4g13930 & gi|20334774 & Putative glycine hydroxymethyltransferase & $51.7 / 7.0$ & 32.1 & 1.9 \\
\hline 59 & Atlg20630 & gi|2511725 & Catalase 1 & $56.8 / 7.4$ & 60.4 & 2.2 \\
\hline 60 & At3g07850 & gi|6648197 & Exopolygalacturonase & $45.6 / 9.7$ & 65.3 & 2.4 \\
\hline 61 & At3g07850 & gi|6648197 & Exopolygalacturonase & $45.6 / 9.7$ & 66.7 & 2.4 \\
\hline 62 & Atlg07920 & gi|295789 & Elongation factor $1-\alpha$ & $49.5 / 9.8$ & 35.2 & 2.4 \\
\hline 63 & At5g03630 & gi|22655344 & Putative monodehydroascorbate reductase & $47.5 / 5.1$ & 50.3 & 2.4 \\
\hline 64 & At5g03630 & gi|22655344 & Putative monodehydroascorbate reductase & $47.5 / 5.1$ & 55.2 & 2 \\
\hline 65 & At3g13920 & gi|14594802 & Translation initiation factor $4 \mathrm{~A}-1$ & $41.8 / 8.8$ & 45.8 & 1.8 \\
\hline 66 & Atlg02500 & gi|81647 & $S$-Adenosylmethionine synthetase 1 & $43.1 / 5.4$ & 39.7 & 2.3 \\
\hline 67 & At2g36880 & gi|22137172 & Putative $S$-adenosylmethionine synthetase & $42.5 / 5.7$ & 31.0 & 1.2 \\
\hline 67 & At4g01850 & gi|23308349 & $S$-Adenosylmethionine synthetase 2 & $43.2 / 5.6$ & 36.4 & 1.3 \\
\hline 68 & At2g36880 & gi|22137172 & Putative $S$-adenosylmethionine synthetase & $42.5 / 5.7$ & 75.4 & 2.4 \\
\hline 69 & Atlg65930 & gi|20453235 & Putative isocitrate dehydrogenase & $45.7 / 6.1$ & 41.5 & 2 \\
\hline 70 & Atlg65930 & gi|20453235 & Putative isocitrate dehydrogenase & $45.7 / 6.1$ & 55.1 & 2.1 \\
\hline 71 & At2g35840 & gi|11127757 & Sucrose-phosphatase 1 & $47.6 / 6.2$ & 53.3 & 2.3 \\
\hline 72 & At4g02930 & gi|31376381 & Putative elongation factor $\mathrm{Tu}$ & $49.4 / 6.3$ & 69.6 & 2.4 \\
\hline 73 & At5g07440 & gi|7576182 & Glutamate dehydrogenase 2 & $44.7 / 6.1$ & 51.1 & 2.2 \\
\hline 74 & At2g33150 & gi|13194830 & Putative acetyl-CoA C-acyltransferase & $48.5 / 9.5$ & 71.4 & 2.1 \\
\hline 75 & At2g37620 & gi|71633 & Actin 1 & $41.7 / 5.2$ & 62.9 & 2.2 \\
\hline 75 & At3g53750 & gi|21554576 & Actin 3 & $41.8 / 5.2$ & 62.9 & 2.2 \\
\hline 76 & At2g37620 & gi|71633 & Actin 1 & $41.7 / 5.2$ & 61.0 & 2.3 \\
\hline 76 & At3g53750 & gi|21554576 & Actin 3 & $41.8 / 5.2$ & 62.9 & 2.2 \\
\hline 77 & At5g59370 & gi|8885543 & Actin 4 & $41.8 / 5.3$ & 38.2 & 1.9 \\
\hline 77 & At3g46520 & gi|28827524 & Actin 12 & $41.8 / 5.3$ & 38.2 & 1.9 \\
\hline 78 & Atlg79550 & gi|30725646 & Putative phosphoglycerate kinase & $42.1 / 5.4$ & 74.3 & 1.6 \\
\hline 79 & At2g20420 & gi|22136422 & Putative succinyl-CoA ligase $\beta$-chain & $45.3 / 6.3$ & 53.7 & 1.7 \\
\hline 80 & At5g16510 & gi|21280929 & Putative reversibly glycosylated polypeptide & $38.6 / 4.9$ & 48.9 & 2.4 \\
\hline 81 & Atlg35720 & gi|12083278 & Annexin 1 & $36.2 / 5.1$ & 79.2 & 1.6 \\
\hline 82 & At3g09820 & gi|6681336 & Adenosine kinase 1 & $37.8 / 5.2$ & 76.7 & 2.2 \\
\hline 83 & At3g02230 & gi|31711848 & Reversibly glycosylated polypeptide- 1 & $40.6 / 5.5$ & 78.7 & 2.4 \\
\hline 84 & At5g15650 & gi|21464559 & Reversibly glycosylated polypeptide-2 & $40.9 / 5.7$ & 79.7 & 2.4 \\
\hline 85 & At2g47470 & gi|24417274 & Disulfide isomerase-like & $39.5 / 5.7$ & 56.5 & 2.3 \\
\hline 86 & At3g17940 & gi|9294498 & Aldose 1-epimerase family protein & $37.2 / 5.9$ & 67.2 & 1.3 \\
\hline 87 & At3g52930 & gi|21592946 & Putative fructose-bisphosphate aldolase & $38.5 / 6.0$ & 43.6 & 2.1 \\
\hline 88 & At3g52930 & gi|21592946 & Putative fructose-bisphosphate aldolase & $38.5 / 6.0$ & 80.2 & 2.4 \\
\hline 89 & At2g30970 & gi|22136256 & Aspartate aminotransferase & $47.7 / 9.1$ & 62.3 & 2.3 \\
\hline 90 & At5g61720 & gi|30793953 & Expressed protein, $A$. thaliana & $43.1 / 8.6$ & 51.8 & 2 \\
\hline 91 & Atlg 26480 & gi|30017291 & 14-3-3 protein GF14 iota & $30.5 / 4.7$ & 63.4 & 2.4 \\
\hline 92 & At3g59480 & gi|6996284 & pfkB-type carbohydrate kinase family protein & $35.0 / 5.1$ & 73.0 & 2.2 \\
\hline
\end{tabular}

\begin{tabular}{|c|c|c|c|c|}
\hline 263 & cytp. & transport & 3 & undeter. \\
\hline 188 & nucleus & prot. proc. & 3 & A \\
\hline 212 & endomb. & unc. & 3 & $P$ \\
\hline 195 & endomb. & energy & 3 & A \\
\hline 222 & unknown & energy & no data & no data \\
\hline 318 & cytoskel & cell fate & 1 & $\mathrm{P}$ \\
\hline 329 & cytoskel & cytosk. & 2 & $\mathrm{P}$ \\
\hline 103 & endomb. & unc. & 3 & $\mathrm{P}$ \\
\hline 338 & cytp. & metaC & 3 & undeter. \\
\hline 272 & endomb. & metaC & 1 & $\mathrm{P}$ \\
\hline 302 & endomb. & metaC & 1 & $P$ \\
\hline 64 & endomb. & unc. & 2 & $\mathrm{P}$ \\
\hline 92 & cytp. & metabolism & no data & no data \\
\hline 177 & endomb. & energy & no data & no data \\
\hline 92 & cytp. & metabolism & no data & no data \\
\hline 355 & cytp. & defense & 2 & $\mathrm{P}$ \\
\hline 267 & endomb. & unc. & 1 & $P$ \\
\hline 277 & endomb. & unc. & 1 & $\mathrm{P}$ \\
\hline 130 & cytp. & prot. synth. & no data & no data \\
\hline 209 & cytp. & metabolism & 4 & A \\
\hline 199 & cytp. & metabolism & 4 & A \\
\hline 142 & unknown & cell fate & 1 & $\mathrm{P}$ \\
\hline 146 & cytp. & metaC & 3 & $P$ \\
\hline 140 & cytp. & metaC & 3 & $\mathrm{P}$ \\
\hline 142 & cytp. & metaC & 3 & $P$ \\
\hline 382 & cytp. & metaC & 3 & $\mathrm{P}$ \\
\hline 161 & endomb. & energy & 3 & $P$ \\
\hline 203 & endomb. & energy & 3 & $\mathrm{P}$ \\
\hline 206 & endomb. & energy & 3 & undeter. \\
\hline 341 & endomb. & prot. synth. & 3 & undeter. \\
\hline 181 & endomb. & metabolism & 2 & $\mathrm{P}$ \\
\hline 245 & cytp. & energy & 3 & $\mathrm{P}$ \\
\hline 295 & cytoskel & cytosk. & 1 & $P$ \\
\hline 295 & cytoskel & cytosk. & 1 & $\mathrm{P}$ \\
\hline 274 & cytoskel & cytosk. & 1 & $P$ \\
\hline 295 & cytoskel & cytosk. & 1 & $\mathrm{P}$ \\
\hline 164 & cytoskel & cytosk. & 1 & $P$ \\
\hline 164 & cytoskel & cytosk. & 1 & $\mathrm{P}$ \\
\hline 263 & cytp. & metaC & 3 & undeter. \\
\hline 148 & endomb. & unc. & 3 & $\mathrm{P}$ \\
\hline 200 & endomb. & metabolism & 1 & $P$ \\
\hline 222 & cytp. & unc. & 3 & $P$ \\
\hline 297 & cytp. & metabolism & 3 & $P$ \\
\hline 303 & endomb. & cell wall & 1 & $\mathrm{P}$ \\
\hline 272 & endomb. & cell wall & no data & no data \\
\hline 153 & endomb. & unc. & 3 & undeter. \\
\hline 162 & cytp. & unc. & 1 & $\mathrm{P}$ \\
\hline 102 & cytp. & metaC & 3 & undeter. \\
\hline 286 & cytp. & metaC & 3 & undeter. \\
\hline 296 & endomb. & metabolism & 2 & undeter. \\
\hline 228 & endomb. & unc. & 1 & $\mathrm{P}$ \\
\hline 232 & cytp. & prot. proc. & 1 & $\mathrm{P}$ \\
\hline 273 & cytp. & energy & 1 & A \\
\hline
\end{tabular}


Table 1 (continued)

\begin{tabular}{|c|c|c|c|c|c|c|c|c|c|c|c|}
\hline Spot No. ${ }^{a}$ & AGI name & Gene index & Protein identity (TAIR description) & $\mathrm{MM} / \mathrm{p} I^{\mathrm{b}}$ & $\begin{array}{l}\text { Sequence } \\
\text { coverage }^{c}\end{array}$ & ProFound-score $^{\mathrm{d}}$ & Mascot-score $^{e}$ & $\begin{array}{l}\text { Subcellular } \\
\text { localization }^{\mathrm{f}}\end{array}$ & $\begin{array}{l}\text { Functional } \\
\text { category }^{\mathrm{g}}\end{array}$ & Genevestigator $^{\mathrm{h}}$ & $\begin{array}{l}\text { Micro array } \\
\text { detection }^{\mathrm{i}}\end{array}$ \\
\hline 93 & At3g59480 & gi|6996284 & pfkB-type carbohydrate kinase family protein & $35.0 / 5.1$ & 65.0 & 2.4 & 294 & cytp. & energy & 1 & A \\
\hline 94 & At2g31390 & gi|22136070 & pfkB-type carbohydrate kinase family protein & $35.2 / 5.2$ & 46.5 & 2.2 & 176 & cytp. & energy & 3 & $\mathrm{P}$ \\
\hline 95 & Atlg07750 & gi|24111337 & Cupin family protein & $38.3 / 5.8$ & 41.9 & 2.3 & 174 & cytp. & unc. & 1 & undeter. \\
\hline 96 & Atlg07750 & gi|24111337 & Cupin family protein & $38.3 / 5.8$ & 48.0 & 2.1 & 170 & cytp. & unc. & 1 & undeter. \\
\hline 97 & At5g43330 & gi|20259605 & Putative malate dehydrogenase & $35.7 / 6.4$ & 68.4 & 2.4 & 297 & cytp. & energy & 1 & $\mathrm{P}$ \\
\hline 98 & Atlg69940 & gi|12325236 & Pectinesterase family protein & $36.6 / 9.3$ & 51.5 & 2.2 & 207 & cell wall & cell wall & 1 & $\mathrm{P}$ \\
\hline 99 & At5g01410 & gi|20453411 & Putative stress-responsive protein & $33.2 / 5.7$ & 17.5 & 1.1 & 64 & cytp. & metabolism & 3 & $\mathrm{P}$ \\
\hline 100 & At5g01410 & gi|20453411 & Putative stress-responsive protein & $33.2 / 5.7$ & 41.4 & 1.9 & 158 & cytp. & metabolism & 3 & $\mathrm{P}$ \\
\hline 101 & Atlg63000 & gi|14423536 & Expressed protein, A. thaliana & $33.6 / 5.8$ & 33.9 & 2.4 & 104 & unknown & unc. & 2 & $\mathrm{P}$ \\
\hline 102 & At2g28680 & gi|4580389 & Cupin family protein & $38.4 / 6.3$ & 65.7 & 2.4 & 266 & cytp. & unc. & 1 & undeter. \\
\hline 103 & At4g10260 & gi|4538955 & pfkB-type carbohydrate kinase family protein & $34.7 / 7.7$ & 58.3 & 2.3 & 169 & cytp. & energy & 1 & A \\
\hline 104 & At4g10260 & gi|4538955 & pfkB-type carbohydrate kinase family protein & $34.7 / 7.7$ & 65.4 & 2.1 & 270 & cytp. & energy & 1 & A \\
\hline 105 & Atlg64980 & gi|34146814 & Expressed protein, $A$. thaliana & $30.2 / 5.3$ & 41.7 & 1.8 & 114 & cytp. & unc. & 1 & $\mathrm{P}$ \\
\hline 106 & Atlg11840 & gi|15810219 & Putative lactoylglutathione lyase & $31.9 / 4.9$ & 52.3 & 2.4 & 189 & unknown & unc. & 3 & $\mathrm{P}$ \\
\hline 107 & Atlg11840 & gi|15810219 & Putative lactoylglutathione lyase & $31.9 / 4.9$ & 30.4 & 2.0 & 82 & unknown & unc. & 3 & $\mathrm{P}$ \\
\hline 108 & Atlg11840 & gi|15810219 & Putative lactoylglutathione lyase & $31.9 / 4.9$ & 52.3 & 2.0 & 154 & unknown & unc. & 3 & $\mathrm{P}$ \\
\hline 109 & At5g04180 & gi|21618128 & Carbonic anhydrase family protein & $31.4 / 7.9$ & 43.3 & 2.3 & 182 & endomb. & unc. & 1 & $\mathrm{P}$ \\
\hline 110 & At5g19510 & gi|15810631 & Elongation factor 1B $\alpha$-subunit 2 & 24.2/4.3 & 45.5 & 2.3 & 149 & ribosome & prot. synth. & 3 & $\mathrm{P}$ \\
\hline 111 & Atlg78300 & gi|21618266 & $14-3-3$ protein GF14 $\omega$ & 29.1/4.6 & 83.0 & 1.5 & 222 & cytp. & prot & 1 & $\mathrm{P}$ \\
\hline 112 & Atlg23730 & gi|21386933 & Putative carbonic anhydrase & $28.8 / 6.7$ & 67.4 & 2.4 & 231 & cytp. & unc. & 1 & undeter. \\
\hline 113 & Atlg47260 & gi|21280965 & $\begin{array}{l}\text { Bacterial transferase hexapeptide } \\
\text { repeat-containing protein }\end{array}$ & $30.0 / 6.9$ & 58.3 & 1.4 & 157 & endomb. & unc. & 3 & $\mathrm{P}$ \\
\hline 113 & At5g20080 & gi|21592883 & Putative NADH-cytochrome $b 5$ reductase & $36.0 / 9.3$ & 57.3 & 1.7 & 172 & endomb. & energy & 3 & A \\
\hline 114 & At2g21870 & gi|19310625 & Expressed protein, $A$. thaliana & $27.6 / 6.3$ & 60 & 2.4 & 150 & endomb. & energy & 3 & $\mathrm{P}$ \\
\hline 115 & At3g55440 & gi|11270444 & Putative triosephosphate isomerase & $27.1 / 5.1$ & 93.3 & 2.1 & 272 & cytp. & metaC & 3 & undeter. \\
\hline 116 & At2g46860 & gi|20197320 & Putative inorganic pyrophosphatase & $24.9 / 5.5$ & 40.7 & 0.8 & 75 & cytp. & metabolism & 1 & $\mathrm{P}$ \\
\hline 117 & Atlg07890 & gi|21554322 & L-Ascorbate peroxidase 1 & $27.5 / 5.7$ & 48.8 & 2.3 & 124 & endomb. & defense & 3 & undeter. \\
\hline 118 & At1g07890 & gi|21554322 & L-Ascorbate peroxidase 1 & $27.5 / 5.7$ & 74.4 & 2.4 & 252 & endomb. & defense & 3 & undeter. \\
\hline 119 & At5g01600 & gi|12642862 & Ferritin 1 & $28.2 / 5.7$ & 35.7 & 2.1 & 121 & endomb. & transport & 4 & $\mathrm{P}$ \\
\hline 120 & At5g20720 & gi|17065646 & $20 \mathrm{kDa}$ chaperonin & 26.8/9.3 & 69.6 & 2.3 & 181 & endomb. & transport & 4 & A \\
\hline 121 & At3g60180 & gi|2497486 & Putative uridylate kinase & $22.5 / 5.7$ & 89.1 & 2.3 & 263 & cytp. & metabolism & 4 & undeter. \\
\hline 122 & At2g47730 & gi|20197312 & Glutathione $S$-transferase 6 & $24.1 / 6.1$ & 26.0 & 1.2 & 69 & endomb. & metabolism & 2 & $\mathrm{P}$ \\
\hline 123 & At2g47730 & gi|20197312 & Glutathione $S$-transferase 6 & $24.1 / 6.1$ & 82.8 & 2.4 & 232 & endomb. & metabolism & 2 & $\mathrm{P}$ \\
\hline 124 & At3g05930 & gi|6714406 & Germin-like protein & 23.0/9.6 & 58.9 & 2.3 & 148 & endomb. & defense & 1 & $\mathrm{P}$ \\
\hline 125 & At3g 52300 & gi|21555349 & ATP synthase D chain-related & $19.6 / 4.9$ & 80.4 & 2.3 & 210 & cytp. & energy & 3 & $\mathrm{P}$ \\
\hline 126 & At4g24640 & gi|21593482 & $\begin{array}{l}\text { Invertase/pectin methylesterase } \\
\text { inhibitor family protein }\end{array}$ & $19.9 / 5.5$ & 77.5 & 2.4 & 280 & endomb. & unc. & 1 & $\mathrm{P}$ \\
\hline 127 & At4g11600 & gi|21617919 & Putative glutathione peroxidase & $18.6 / 7.4$ & 66.9 & 2.4 & 162 & endomb. & defense & 2 & $\mathrm{P}$ \\
\hline 128 & At3g06050 & gi|20466103 & Alkyl hydroperoxide reductase & 21.4/9.6 & 65.2 & 2.4 & 199 & endomb. & unc. & 2 & $\mathrm{P}$ \\
\hline 129 & At2g21130 & gi|21593051 & Peptidyl-prolyl cis-trans isomerase/cyclophilin & $18.5 / 9.4$ & 82.8 & 2.4 & 196 & cytp. & prot. proc. & 2 & $\mathrm{P}$ \\
\hline 129 & At4g38740 & gi|21593682 & Peptidyl-prolyl cis-trans isomerase/cyclophilin & $18.4 / 9.0$ & 77.9 & 2.1 & 180 & cytp. & prot. proc. & 3 & $\mathrm{P}$ \\
\hline 130 & At2g21130 & gi|21593051 & Peptidyl-prolyl cis-trans isomerase/cyclophilin & $18.5 / 9.4$ & 90.2 & 2.1 & 201 & cytp. & prot. proc. & 2 & $\mathrm{P}$ \\
\hline 131 & At2g21130 & gi|21593051 & Peptidyl-prolyl cis-trans isomerase/cyclophilin & $18.5 / 9.4$ & 90.2 & 1.9 & 198 & cytp. & prot. proc. & 2 & $\mathrm{P}$ \\
\hline $132^{*}$ & At3g07850 & gi|11994377 & Exopolygalacturonase & $45.7 / 9.7$ & 31.0 & 2.3 & 156 & endomb. & unc. & 1 & $\mathrm{P}$ \\
\hline $133^{*}$ & At3g07850 & gi|6648197 & Exopolygalacturonase & $45.6 / 9.7$ & 30.9 & 2.3 & 136 & endomb. & unc. & 1 & $\mathrm{P}$ \\
\hline 134 & At5g52360 & gi|10177402 & Putative actin-depolymerizing factor & $15.3 / 5.4$ & 78.5 & 2.3 & 125 & cytp. & cell wall & 1 & $P$ \\
\hline 135 & At4g29340 & gi|21537389 & Profilin 3 & $14.4 / 4.9$ & 57.5 & 2.0 & 114 & cytp. & cytosk. & 1 & $\mathrm{P}$ \\
\hline $136^{*}$ & At4g29340 & gi|21537389 & Profilin 3 & $14.4 / 4.9$ & 44.0 & 1.9 & 82 & cytp. & cytosk. & 1 & $\mathrm{P}$ \\
\hline 137 & Atlg15415 & gi|5103841 & Expressed protein (low similarity to LEA protein) & $10.4 / 5.9$ & 76.0 & 2.4 & 137 & nucleus & unc. & no data & no data \\
\hline 138 & At4g13560 & gi|28827234 & LEA domain-containing protein & $11.6 / 7.7$ & 88.1 & 2.4 & 178 & nucleus & unc. & 1 & $\mathrm{P}$ \\
\hline 139 & At4g13560 & gi|28827234 & LEA domain-containing protein & $11.6 / 7.7$ & 62.4 & 2.3 & 106 & nucleus & unc. & 1 & $\mathrm{P}$ \\
\hline 140 & At5g12140 & gi|21593904 & Putative cysteine protease inhibitor & $11.2 / 4.9$ & 58.4 & 0.7 & 64 & cytp. & unc. & 3 & $\mathrm{P}$ \\
\hline 141 & At3g51030 & gi|21617958 & Thioredoxin H-type 1 & $12.7 / 5.6$ & 48.2 & 0.7 & 68 & cytp. & unc. & 1 & $\mathrm{P}$ \\
\hline
\end{tabular}




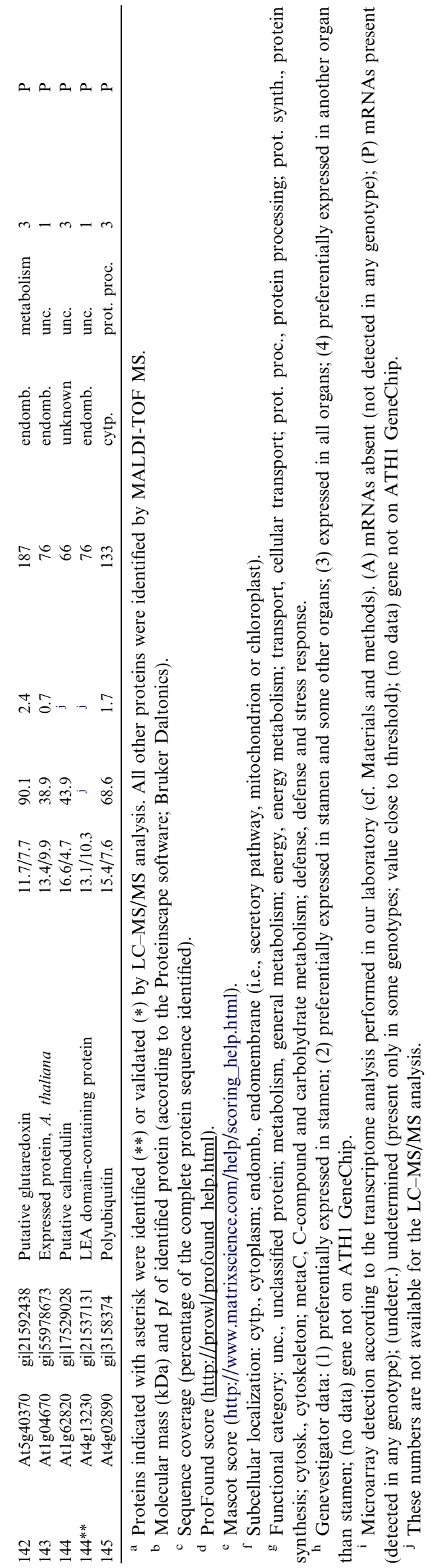

\section{Protein identification by PMF analysis}

All protein spots were processed by automated in-gel tryptic digestion and MALDI-TOF MS analysis to generate PMFs. Spots with non-significant identification scores were further analyzed by LC-MS/MS. The 155 spectra obtained were used to screen the NCBI database, which enabled us to assign putative identity to 145 spots representing 121 different proteins (Table 1). For 10 of the 145 spots analyzed, two distinct polypeptides were identified either by MALDI-TOF MS or by LC-MS/MS. In addition, LC-MS/MS revealed the identity of 4 further proteins (Table 1). Identified proteins ranged in calculated molecular mass from 10.4 to $108 \mathrm{kDa}$ and in calculated $\mathrm{p} I$ from 4.2 to 9.8 (Table 1). The average deviations of the measured peptide masses compared to the theoretical values of the respective peptides of the identified proteins were less than $0.1 \mathrm{Da}$. The identity of 105 spots was assigned in at least two replicates of different genotypes, 40 others were characterized in at least two replicates of the same genotype. The low complexity of the male gametophyte and the relative purity of the pollen samples appear to result in a beneficial signal-to-noise ratio which may explain the highly significant Mascot and ProFound scores obtained in our analysis (Table 1). All identified proteins were designated with arbitrary spot numbers as shown in Fig. 1. As indicated in Table 1, all Gene Index (GI) annotations could be assigned with the corresponding AGI name [8] and the designation of the corresponding genes is given according to the TAIR database (http://www.arabidopsis.org/index.jsp). The predicted molecular masses and isoelectric points for the majority of the identified proteins were consistent with the experimental data as judged from the location of the respective polypeptide spots within the $2 \mathrm{D}$ gels. This further corroborated the identity of the recognized polypeptides. Exceptions included spot 1 (Atlg49490) which exhibits a higher molecular mass $(\sim 150 \mathrm{kDa})$ than calculated $(90.9 \mathrm{kDa})$. Indeed, big proteins can display a strong tendency to aggregate, complicating migration. Conversely, products related to spots 132 and 133 (At3g07849) show a low molecular mass $(\sim 17 \mathrm{kDa})$ compared with the calculated value $(\sim 45 \mathrm{kDa})$. This could result from post-translational modifications or degradation of intact proteins during the extraction procedure.

\section{Presence of multiple polypeptide isoforms}

Generally, proteins can be assigned to two classes of isoforms. The first class comprises polypeptide variants encoded by the same gene (e.g., splice variants or different post-translational modifications). The second class encompasses highly sequence-related protein isoforms encoded by distinct genes. In our study, 27 candidates of the first class of isoforms were detected and 16 of the second. Approximately $44 \%$ of all isoforms likely represent different phosphorylation states since they are characterized by the same apparent molecular mass but different $\mathrm{p} I$ values. As 
suggested by the previous analysis from rice anthers about proteins present at different stages of pollen development [16], occurrence of multiple isoforms originating from a single gene could indicate that post-translational modifications might play a significant role during pollen development.

\section{Subcellular localization}

The potential subcellular localization of identified polypeptides was assigned using web-based tools as described in Materials and methods. Despite a somewhat different classification, the observed proportions were compared to the subcellular localization prediction of the whole Arabidopsis protein set [26]. We noted that the largest fraction ( 41\%) of the identified proteins appears to be cytoplasmic which is consistent with the respective value for the whole Arabidopsis proteome $(58 \%)$. A second significant proportion $(\sim 40 \%)$ is potentially targeted to the endomembrane system which is also similar to the respective fraction of the total Arabidopsis protein set (43\%). Furthermore, approximately $4 \%$ of the pollen polypeptides are predicted to reside in the nucleus, $\sim 5 \%$ are presumably associated with the cytoskeleton, $\sim 1.6 \%$ reside in the vacuole, $\sim 0.8 \%$ in the cell wall, $\sim 0.8 \%$ in the ribosome, and the localization of $\sim 7 \%$ of the proteins cannot currently be predicted. Notably, none of the Arabidopsis pollen coat proteins described in the study of Mayfield et al. [17] was identified in our analysis. The extreme hydrophobic properties of these proteins could mean that they were not extracted in our experimental conditions. Besides, only 6 proteins with predicted transmembrane domains (At2g07698, At1g09210, At2g47470, At1g69940, At1g56340, and At3g07850) were identified. This may relate to the fact that generally integral membrane proteins, in particular those with multiple membrane-spanning domains, do not resolve well during isoelectric focusing and are therefore inherently underrepresented in 2-DE [27]. This fact represents a well-known limitation of 2-DE-based proteomic approaches that also applies to our study.

\section{Functional categories}

We determined the predicted functional categories assigned to the identified pollen proteins. The majority of the polypeptides are involved in metabolism $(\sim 42 \%)$. This could potentially reflect a special requirement of the mature pollen, since only $22.5 \%$ of the total proteome of $\mathrm{Ara}$ bidopsis is dedicated to this function [8]. Alternatively, since metabolic enzymes are generally highly abundant, soluble proteins, this functional category might be overrepresented in our analysis. The latter hypothesis appears more likely since only $\sim 19 \%$ of the pollen transcriptome encodes metabolic proteins (see below; [10]). In detail, $\sim 19 \%$ of the identified proteins implicated in metabolic functions are related to carbohydrate metabolism (e.g., triosephosphate isomerase -At3g55440-, glycoside hydrolase -At3g5440-, glucose dehydrogenase -At5g15490-, or fruc- tose-bisphospate aldolase -At3g52930) and 51\% to energy metabolism. Similar to the Arabidopsis total proteome analysis (i.e., 11.7\%) [8], 9\% of the Arabidopsis mature pollen proteome is implicated in biogenesis of cellular compounds. With respect to this function, enzymes required for the synthesis of cell wall constituents, such as pectinesterase -Atlg69940- or exopolygalacturonase At4g24640, At3g07850- have been identified. Cytoskeleton-associated proteins have also been found (e.g., actin -At2g37620, At3g53750, At3g46520, At5g59370-, profilin -At4g29340-, and tubulin -At1g50010, At5g44340). The latter polypeptides are presumably involved in the regulation of polarized tip-growth of pollen tubes upon germination and along the female reproductive tract [7]. In addition, more than $9 \%$ of the identified proteins exhibit a protein processing function (14\% in the Arabidopsis total proteome [8]). Interestingly, the majority of these proteins $(75 \%)$ are preferentially implicated in the determination of protein fate rather than in protein synthesis. This is consistent with the idea that Arabidopsis mature pollen is charged with a pre-formed translational apparatus enabling rapid activation upon hydration and germination [10]. A considerable number $(27 \%$, i.e., 33 proteins $)$ of the experimentally identified polypeptides are of unknown function. This number is essentially in accordance with the respective transcriptome data (see below). A large set of unclassified proteins was also found in recent plant, human, and yeast mitochondrial proteome analyses [28-30] and interpreted as an indication for a putative wealth of as yet undiscovered mitochondrial functions. By analogy, presence of a major set of proteins with unknown functions in pollen may hint at as yet unidentified cellular processes, of which some might be specific for the male gametophyte. The latter hypothesis is supported by the fact that 16 of the respective genes are preferentially expressed in the stamen (Genevestigator data). The identification of these unclassified pollen proteins provides a basis for the use of reverse genetics to identify novel biological functions in plants by taking advantage of the reduced complexity of the pollen system.

\section{Comparison with pollen transcriptome data}

Expression data of genes corresponding to proteins identified in our study were investigated by using the microarray data deposited at the Genevestigator server [24]. This revealed that a relative majority of these genes $(40 \%)$ are expressed in all organs. Approximately $48 \%$ of this group are implicated in metabolic functions, suggesting that they act in general plant-wide housekeeping. A good proportion $(30.5 \%)$ of our candidates are preferentially expressed in the stamen organ and $16.5 \%$ are preferentially expressed in stamen and some other organs. Only a minority of the candidates $(5 \%)$ are preferentially expressed in another organ than stamen.

Presence of proteins identified in our study was compared to data obtained by a previous transcriptome 
analysis of mature pollen [10]. In terms of functional categories, general metabolism $(20.6 \%)$, protein processing $(9 \%)$, and stress-related (4\%) functions assigned on the basis of our proteomic approach reflect similar proportions, namely $18 \%, 6 \%$, and $6 \%$, respectively, in the microarraybased transcriptome study. Polypeptides devoted to cellular transport $(4 \%)$ and signaling $(0 \%)$ functions appear underrepresented in our proteome analysis compared to gene expression data ( $8 \%$ and $13 \%$, respectively). Complete absence of proteins implicated in cellular signaling may be due to the fact that many of these polypeptides are either of low abundance and/or integral membrane proteins that are difficult to detect in 2-DE-based approach. Low abundance may also account for a similar discrepancy with regard to transcriptional regulators $(0 \%$ in proteome versus $7 \%$ in transcriptome). Surprisingly, products exhibiting a function in energy metabolism appear to be highly represented in the proteome $(21 \%)$ compared to the transcriptome $(1 \%)$. This may indicate that mature pollen is well prepared with pre-existing metabolic enzymes for rapid energy conversion upon germination and pollen tube expansion. Both our proteomic approach and the global analysis of the pollen transcriptome revealed a high proportion of as yet unclassified proteins ( $27 \%$ and $19 \%$, respectively; see above).

Besides the above-described global comparison, we compared each identified protein with its respective transcriptome data. The latter either originate from experiments performed in our laboratory (cf. Materials and methods) or publicly available datasets ([12] and unpublished datasets available at the NASC website; http://affymetrix.arabidopsis.info/narrays/experimentbrowse.pl; slides Honys_MPG1_SLD; ATGE_73A and DT001_AG_pollen, respectively). In total, transcripts of proteins unequivocally identified in 14 spots (corresponding to 10 distinct AGI loci) have been reproducibly classified as absent (based on MicroArray Suite 5.0 software) in our own transcriptome analyses (At2g07698; At2g33210; At3g18190; At3g23990; At3g59480; At4g10260; At5g03630; At5g20080; At5g20720; At5g42740, see Table 1; please note that our transcriptome studies were carried out by applying the same plant growth conditions and techniques for pollen mining as for the proteome analysis). Likewise, nine of the ten transcripts were declared as absent in the study of Pina et al. [12], while the remaining datasets deposited on the NASC webpage provide ambiguous results for these proteins. The latter might be due to differences in plant growth and/or pollen mining as compared to our techniques. In summary, the respective transcripts might either be absent in mature pollen or present at very low levels. This observation implies that low transcript abundance may suffice to ensure accumulation of a significant proportion of protein in some instances (e.g., for proteins with a low turnover rate). Alternatively, the respective polypeptides might have been translated at an earlier stage of pollen development (e.g., in the bicellular stage) and still persist in mature pollen. In any case, this finding further underlines the importance of proteome studies as valuable supplements to transcriptome analyses.

\section{Acknowledgments}

We acknowledge the SALK Institute (La Jolla, USA), Syngenta Biotechnology Inc. (Research Triangle Park, USA), and the Sainsbury Laboratory (Norwich UK) for providing Arabidopsis knockout lines either directly to us or via the NASC repository. This work was supported by grants of the Max-Planck society to R.P. and J.S.

\section{References}

[1] S. McCormick, Male gametophyte development, Plant Cell 5 (1993) 1265-1275.

[2] D. Twell, S.K. Park, E. Lalanne, Asymmetric division and cell-fate determination in developing pollen, Trends Plant Sci. 3 (1998) 305310 .

[3] J.P. Mascarenhas, Molecular mechanisms of pollen tube growth and differentiation, Plant Cell 5 (1993) 1303-1314.

[4] S. McCormick, Control of male gametophyte development, Plant Cell 16 (2004) S142-S153.

[5] J.A. Feijó, J. Sainhas, T. Holdaway-Clarke, M.S. Cordeiro, J.G. Kunkel, P.K. Hepler, Cellular oscillations and the regulation of growth: the pollen tube paradigm, BioEssays 23 (2001) 86-94.

[6] J.A. Feijó, S.S. Costa, A.M. Prado, J.D. Becker, A.C. Certa, Signaling by tips, Curr. Opin. Plant Biol. 7 (2004) 589-598.

[7] K. Hepler, L. Vidali, A.Y. Cheung, Polarized cell growth in higher plants, Annu. Rev. Cell Dev. Biol. 17 (2001) 159-187.

[8] Arabidopsis Genome Initiative, Analysis of the genome sequence of the flowering plant Arabidopsis thaliana, Nature 408 (2000) 796815.

[9] J.D. Becker, L.C. Boavida, J. Carneiro, M. Haury, J.A. Feijó, Transcriptional profiling of Arabidopsis tissues reveals the unique characteristics of the pollen transcriptome, Plant Physiol. 133 (2003) 713-725.

[10] D. Honys, D. Twell, Comparative analysis of the Arabidopsis pollen transcriptome, Plant Physiol. 132 (2003) 640-652.

[11] D. Honys, D. Twell, Transcriptome analysis of haploid male gametophyte development in Arabidopsis, Genome Biol. 5 (2004) R85.

[12] C. Pina, F. Pinto, J.A. Feijó, J.D. Becker, Gene family analysis of the Arabidopsis pollen transcriptome reveals biological implications for cell growth, division control, and gene expression regulation, Plant Physiol. 138 (2005) 744-756.

[13] J. Cordewener, J. Bergervoet, C.M. Liu, Changes in protein synthesis and phosphorylation during microspore embryogenesis in Brassica napus, J. Plant Physiol. 156 (2000) 156-163.

[14] A. Kalinowski, K. Winiarczyk, B. Wojciechowska, Pollen proteins after two-dimensional gel electrophoresis and pollen morphology of the amphiploids Aegilops kotschyi and Ae. variabilis with Secale cereale, Sex, Plant Reprod. 14 (2001) 153-161.

[15] N. Imin, T. Kerim, J.J. Weinman, B.G. Rolfe, Characterization of rice anther proteins expressed at the young microspore stage, Proteomics 1 (2001) 1149-1161.

[16] T. Kerim, N. Imin, J.J. Weinman, B.G. Rolfe, Proteome analysis of male gametophyte development in rice anthers, Proteomics 3 (2003) $738-751$.

[17] J.A. Mayfield, A. Fiebig, S.E. Johnstone, D. Preuss, Gene families from the Arabidopsis thaliana pollen coat proteome, Science 292 (2001) 2482-2485.

[18] J.M. Alonso, A.N. Stepanova, T.J. Leisse, C.J. Kim, H. Chen, P. Shinn, D.K. Stevenson, J. Zimmerman, P. Barajas, R. Cheuk, C. Gadrinab, C. Heller, A. Jeske, E. Koesema, C.C. Meyers, H. Parker, L. Prednis, Y. Ansari, N. Choy, H. Deen, M. Geralt, N. Hazari, E. Hom, M. Karnes, C. Mulholland, R. Ndubaku, I. Schmidt, P. Guzman, L. Aguilar-Henonin, M. Schmid, D. Weigel, D.E. Carter, T. Marchand, E. Risseeuw, D. Brogden, A. Zeko, W.L. Crosby, C.C. 
Berry, J.R. Ecker, Genome-wide insertional mutagenesis of Arabidopsis thaliana, Science 301 (2003) 653-657.

[19] A. Sessions, E. Burke, G. Presting, G. Aux, J. McElver, D. Patton, B. Dietrich, P. Ho, J. Bacwaden, C. Ko, J. Clarke, D. Cotton, D. Bullis, J. Snell, T. Miguel, D. Hutchison, B. Kimmerly, T. Mitzel, F. Katagiri, J. Glazebrook, M. Law, S. Goff, A high-throughput Arabidopsis reverse genetics system, Plant Cell 14 (2002) 29852994.

[20] A. Tissier, S. Marillonnet, V. Klimyuk, K. Patel, M. Angel Torres, G. Murphy, J.D.G. Jones, Multiple independent defective suppressormutator transposon insertions in Arabidopsis: a tool for functional genomics, Plant Cell 11 (1999) 1841-1852.

[21] S.A. Johnson-Brousseau, S. McCormick, A compendium of methods useful for characterizing Arabidopsis pollen mutants and gametophytically expressed genes, Plant J. 39 (2004) 761-775.

[22] J. Gobom, M. Schuerenberg, M. Mueller, D. Theiss, H. Lehrach, E. Nordhoff, Cyano-4-hydroxycinnamic acid affinity sample preparation. A protocol for MALDI-MS peptide analysis in proteomics, Anal. Chem. 73 (2001) 434 438.

[23] J.C. Redman, B.J. Haas, G. Tanimoto, C.D. Town, Development and evaluation of an Arabidopsis whole genome Affymetrix probe array, Plant J. 38 (2004) 545-561.

[24] P. Zimmermann, M. Hirsch-Hoffmann, L. Hennig, W. Gruissem, GENEVESTIGATOR. Arabidopsis microarray database and analysis toolbox, Plant Physiol. 136 (2004) 2621-2632.
[25] A. Devoto, H.A. Hartmann, P. Piffanelli, C. Elliott, C. Simmons, G. Taramino, C.-S. Goh, F.E. Cohen, B.C. Emerson, P. Schulze-Lefert, R. Panstruga, Molecular phylogeny and evolution of the plant-specific seven transmembrane MLO family, J. Mol. Evol. 56 (2003) 77-88.

[26] R.A. Gutiérrez, P.J. Green, K. Keegstra, J.B. Ohlrogge, Phylogenetic profiling of the Arabidopsis thaliana proteome: what proteins distinguish plants from other organisms? Genome Biol. 5 (2004) R53.

[27] N.A. Jones, Two-dimensional polyacrylamide gel electrophoresis for proteome analyses, in: P. Cutler (Ed.), Methods in Molecular Biology, vol. 244, Humana Press, Totowa, NJ (USA), 2004, pp. 353-359.

[28] J.L. Heazlewood, J.S. Tonti-Filippini, A.M. Gout, D.A. Day, J. Whelan, A.H. Millar, Experimental analysis of the Arabidopsis mitochondrial proteome highlights signaling and regulatory components, provides assessment of targeting prediction programs, and indicates plant-specific mitochondrial proteins, Plant Cell 16 (2004) 241-256.

[29] S.W. Taylor, E. Fahy, B. Zhang, G.M. Glenn, D.E. Warnock, S. Wiley, A.N. Murphy, S.P. Gaucher, R.A. Capaldi, B.W. Gibson, S.S. Ghosh, Characterization of the human heart mitochondrial proteome, Nat. Biotechnol. 21 (2003) 281-286.

[30] A. Sickmann, J. Reinders, Y. Wagner, C. Joppich, R. Zahedi, H. Meyer, B. Schonfisch, I. Perschil, A. Chacinska, B. Guiard, P. Rehling, N. Pfanner, C. Meisinger, The proteome of Saccharomyces cerevisiae mitochondria, Proc. Natl. Acad. Sci. USA 100 (2003) 13207-13212. 